International Journal of Pure and Applied Mathematics

Volume 103 No. 1 2015, 61-70

ISSN: 1311-8080 (printed version); ISSN: 1314-3395 (on-line version)

url: http://www.ijpam.eu

doi: http://dx.doi.org/10.12732/ijpam.v103i1.5

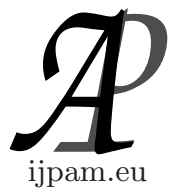

\title{
A MEASURE OF GLOBAL EFFICIENCY IN NETWORKS
}

\author{
Aysun Aytac ${ }^{1}$, Betul Atay ${ }^{2}$ \\ ${ }^{1,2}$ Department of Mathematics \\ Faculty of Science \\ Ege University \\ 35100, Bornova, Izmir, TURKEY
}

\begin{abstract}
Networks are known to be prone to node or link failures. A central issue in the analysis of complex networks is the assessment of their robustness and vulnerability. A network is usually represented by an undirected simple graph where vertices represent processors and edges represent links between processors. Different approaches to properly define a measure for graph vulnerability has been proposed so far.

The distance $d(i, j)$ between any two vertices $i$ and $j$ in a graph is the number of edges in a shortest path between $i$ and $j$. If there is no path connecting $i$ and $j$, then $d(i, j)=\infty$. Latora and Marchiori introduced the measure of efficiency between vertices in a graph in 2001. The unweighted efficiency between two vertices $i$ and $j$ is defined to be $\varepsilon_{i j=1 / d_{i j}}$ for all $i \neq j$. The global efficiency of a graph $E_{\text {glob }}=\frac{2}{n(n-1)} \sum_{i \neq j} \varepsilon\left(v_{i}, v_{j}\right)$ which is simply the average of the efficiencies over all pairs of distinct $n$ vertices. In this paper, we study the global efficiency of special graphs. The behavior of this distance-related parameter on special graphs is taken into account by graphical analysis.
\end{abstract}

AMS Subject Classification: 05C40, 68M10, 68R10

Key Words: graph vulnerability, network design and communication, efficiency, global efficiency

Received: March 13, 2015

(c) 2015 Academic Publications, Ltd. url: www.acadpubl.eu 


\section{Introduction}

Networks are used for modeling different systems such as chemical systems, neural networks, social systems or the Internet and the World Wide Web. The stability of a communication network, composed of processing nodes and communication links, is of prime importance to network designers.

Vulnerability is a generalized conception. There are a lot of different definitions of vulnerability. Vulnerability is usually portrayed in negative terms as the susceptibility to be harmed. It is degree to which a systemic susceptible to and is unable to cope with adverse effects. In this paper, the vulnerability is defined as the decrease of system efficiency after attack. The vulnerability of a communication network measures the resistance of the network to disruption of operation after the failure of certain stations or communication links. Communication networks must be constructed to be as stable as possible, not only with respect to the initial disruption, but also with respect to the possible reconstruction of the network. If we think of a graph as modeling a network, there have been several proposals for measures of the stability of a communication network including connectivity, toughness, scattering number, binding number and integrity (see [1]-[6]- [9]- [13]).

In 2001, Latora and Marchiori introduced the measure of efficiency between vertices in a graph (see [10]). The vulnerability of a network can be measured by the global efficiency of the graph describing the network. The global efficiency is a measure of the network performance and network efficiency based in the structure or topology of the network by the connections of the graph. The aim of global efficiency is simply to measure the average of the efficiencies over all pairs of the distinct vertices. Consider two different graph having the same graph vulnerability characteristics: their connectivity, toughness, integrity, binding number and scattering number is equal. In such a case, global efficiency recognizes the difference between these two graphs.

In this paper, we consider simple finite undirected graphs without loops and multiple edges. Let $G=(V, E)$ be a graph with a vertex set $V=V(G)$ and an edge set $E=E(G)$. The order of $G$ is the number of vertices in $G$. The distance $d(u, v)$ between two vertices $u$ and $v$ in $G$ is the length of a shortest path between them. If $u$ and $v$ are not connected, then $d(u, v)=\infty$, and for $u=v, d(u, v)=0$. The eccentricity of a vertex $v$ in $G$ is the distance from $v$ to a vertex farthest away from $v$ in $G$. The diameter of $G$, denoted by $\operatorname{diam}(G)$, is the largest distance between two vertices in $V(G)$. The degree $\operatorname{deg}_{G}(v)$ of a vertex $v$ in $V(G)$ is the number of edges incident to $v$. The maximum degree of $G$ is $\Delta(G)=\left\{\max \operatorname{deg}_{G}(v) \mid v \in V(G)\right\} \quad$ (see [4]-[5]-[9]-[12]-[13]). 
The paper proceeds as follows. In Section 2, the definitions of efficiency and global efficiency and known results are given. Section 3 several results on global efficiency of some special graphs. Section 4 concludes the paper.

\section{Efficiency and Global Efficiency}

In 2001, Latora and Marchiori introduced the measure of efficiency between vertices in a graph ( see [10]). The global efficiency is a measure of the network performance and network efficiency based in the structure or topology of the network by the connections of the graph. The efficiency between two vertices $i$ and $j$ is defined to be $\varepsilon(i, j)=\frac{1}{d(i, j)}$ for all $i \neq j$, where $d(i, j)$ is the length of a shortest path between two vertices $i$ and $j$ in $G$, so less distant connections are more valuable than more distant ones. The global efficiency of a graph $E_{\text {glob }}(G)=\frac{2}{n(n-1)} \sum_{i \neq j \in V(G)} \varepsilon(i, j)$ which is simply the average of the efficiencies over all pairs of the distinct vertices.

Note that $0 \leq E_{\text {glob }}(G) \leq 1$ is a normalized measure where its maximum value is reached for the complete graph $K_{n}$ on $n$ nodes. In this sense, the global efficiency is expressed as a percentage of network performance, $\sum_{i \neq j \in V(G)} \varepsilon(i, j)$, of what ideally could be expected, $n(n-1)$, in a complete graph as ideal case.

Since for a given number of nodes, the performance and therefore the global efficiency of the graph increases with the number of edges.

Vulnerability measures the stability and robustness of the global performance of the graph under external perturbations (random or targeted failures or attacks). When vulnerability is defined as the relative drop in the global efficiency $E_{g l o b}(G)$, a negative value of vulnerability can appear (see[2] -[3]-[7][8]-[11]).

As in examples, we consider the two graphs, both of which have same edges and vertices. For the connectivity and the diameter value of two graphs $G_{1}$ and $G_{2}$ the equalities are $k\left(G_{1}\right)=k\left(G_{2}\right)$ and $\operatorname{diam}\left(G_{1}\right)=\operatorname{diam}\left(G_{2}\right)$. According to these statements, we can not say that which graph is more reliable. Then, for measuring the reliability, we compute average global efficiency of these two graphs. If $E_{\text {glob }}\left(G_{1}\right)<E_{\text {glob }}\left(G_{2}\right)$, we can say that graph $G_{2}$ is more reliable than graph $G_{1}$.

Theorem 1. [8] $E_{\text {glob }}\left(P_{n}\right)=\frac{2}{n(n-1} \sum_{i=1}^{n-1} \frac{n-i}{i}$.

Theorem 2. [8] $\lim _{x \rightarrow \infty} E_{\text {glob }}\left(P_{n}\right)=0$. 


\section{Global Efficiency of Some Special Graphs}

In this section, we give some results on the global efficiency of some special graphs are calculated. These graphs are $C_{n}$ cycle graph, $S_{1, n}$ star graph, $W_{1, n}$ wheel graph, $K_{n}$ complete graph.

Theorem 3. If $C_{n}$ is a cycle graph with $n$ vertices, then the global efficiency of the cycle is

$$
E_{\text {glob }}\left(C_{n}\right)=\frac{2}{n-1} \sum_{i=1}^{\lfloor n / 2\rfloor-1} \frac{1}{i}+ \begin{cases}\frac{1}{(n-1)\lfloor n / 2\rfloor}, & \text { if } n \text { is odd } \\ \frac{2}{(n-1)\lfloor n / 2\rfloor}, & \text { if } n \text { is even }\end{cases}
$$

Proof. There exist two cases for the shortest paths between the pairs of vertices according to diameter of the graph.

Case 1 If $d\left(v_{i}, v_{j}\right)<\operatorname{diam}\left(C_{n}\right)$ for $\forall v_{i}, v_{j} \in V\left(C_{n}\right)$, then the length of shortest path in $C_{n}$ is $k$, where $k=\overline{1, \operatorname{diam}\left(C_{n}\right)-1}$. Hence, the value of efficiency of these vertices is $\varepsilon\left(v_{i}, v_{j}\right)=\frac{1}{k}$, for all $v_{i} \neq v_{j}$. Since this value of $\varepsilon(i, j)$ holds for every other the pairs of vertices $C_{n}$, combining the values for $\forall v_{i}, v_{j} \in V\left(C_{n}\right)$ yields

$$
\sum_{k=1}^{\operatorname{diam}\left(C_{n}\right)-1} \varepsilon\left(v_{i}, v_{j}\right)=\sum_{k=1}^{\operatorname{diam}\left(C_{n}\right)-1} \frac{1}{k}
$$

There are $n$ different pairs of vertices in this case. Hence, we have

$$
\sum \sum_{k=1}^{\operatorname{diam}\left(C_{n}\right)-1} \frac{1}{k}=n \sum_{k=1}^{\operatorname{diam}\left(C_{n}\right)-1} \frac{1}{k}
$$

Case 2 If $d\left(v_{i}, v_{j}\right)=\operatorname{diam}\left(C_{n}\right) \forall v_{i}, v_{j} \in V\left(C_{n}\right)$, then we have two cases depending on the vertices of $C_{n}$.

Subcase 1 Let $n$ be odd. Then, we consider the pairs of vertices in such that $d\left(v_{i}, v_{i+\operatorname{diam}\left(C_{n}\right)}\right)=\operatorname{diam}\left(C_{n}\right)$, where $i=\overline{1, n}$, and the $i+\operatorname{diam}\left(C_{n}\right)$ is taken modula $n$. So, the value of efficiency for these vertices is $\varepsilon\left(v_{i}, v_{i+\operatorname{diam}\left(C_{n}\right)}\right)=\frac{1}{\operatorname{diam}\left(C_{n}\right)}$. It is clear that $C_{n}$ has diameter $\left\lfloor\frac{n}{2}\right\rfloor$. There are $n$ different pairs of vertices in this case. Hence, we have,

$$
\sum \varepsilon\left(v_{i}, v_{i+\left\lfloor\frac{n}{2}\right\rfloor}\right)=n \frac{1}{\lfloor n / 2\rfloor} .
$$


By Case 1, Subcase 1 of Case2 and the definition of global efficiency, the following result can be derived easily.

$$
E_{\text {glob }}\left(C_{n}\right)=\frac{2}{n(n-1)}\left(n \sum_{i=1}^{\lfloor n / 2\rfloor-1} \frac{1}{i}+n \frac{1}{\lfloor n / 2\rfloor}\right)
$$

where, $n$ is odd.

Subcase 2 Let $n$ be even. Then, we consider the pairs of vertices in such that $d\left(v_{i}, v_{i+\lfloor n / 2\rfloor}\right)=\lfloor n / 2\rfloor$, where $i=\overline{1, \frac{n}{2}}$, and the $i+\lfloor n / 2\rfloor$ is taken modula $n$. Hence, the value of efficiency is $\varepsilon\left(v_{i}, v_{i+\lfloor n / 2\rfloor}\right)=\frac{1}{\lfloor n / 2\rfloor}$.

Since the value of $\varepsilon(i, j)$ holds for every other $n / 2$ pairs of vertices $C_{n}$, combining the efficiency values for $\forall v_{i}, v_{j} \in V\left(C_{n}\right)$ yields

$$
\sum \varepsilon\left(v_{i}, v_{i+\left\lfloor\frac{n}{2}\right\rfloor}\right)=\frac{n}{2} \frac{1}{\lfloor n / 2\rfloor}
$$

By Case 1, Subcase 2 of Case2 and the definition of global efficiency, the following result can be derived easily.

$$
E_{\text {glob }}\left(C_{n}\right)=\frac{2}{n(n-1)}\left(n \sum_{i=1}^{\lfloor n / 2\rfloor-1} \frac{1}{i}+\frac{n}{2} \frac{1}{\lfloor n / 2\rfloor}\right)
$$

where, $n$ is even.

By summing Case 1 and 2, we obtain,

$$
E_{\text {glob }}\left(C_{n}\right)=\frac{2}{n-1} \sum_{i=1}^{\lfloor n / 2\rfloor-1} \frac{1}{i}+ \begin{cases}\frac{1}{(n-1)\lfloor n / 2\rfloor}, & \text { if } n \text { is odd } \\ \frac{(n-1)\lfloor n / 2\rfloor}{(n)}, & \text { if } \text { is even }\end{cases}
$$

The proof is completed.

Theorem 4. If $K_{n}$ is a complete graph with $n$ vertices, then the global efficiency of the complete graph is $E_{\text {glob }}\left(K_{n}\right)=1$.

Proof. Since in a complete graph all vertices are mutually adjacent, distances between each pair of vertices are 1 , that is, for $\forall v_{i}, v_{j} \in V\left(K_{n}\right), i \neq j$, $i, j=\overline{1, n}, d\left(v_{i}, v_{j}\right)=1$.

By the definition of global efficiency, the following result can be derived easily.

$$
E_{g l o b}\left(K_{n}\right)=\frac{2}{n(n-1)}\left[\left(\begin{array}{l}
n \\
2
\end{array}\right) 1\right]
$$




$$
\begin{aligned}
& =\frac{2}{n(n-1)} \frac{n(n-1)}{2} \\
& =1
\end{aligned}
$$

The proof is completed.

Theorem 5. If $W_{1, n}$ is a wheel graph with $n+1$ vertices, then the global efficiency of the wheel graph is $E_{\text {glob }}\left(W_{1, n}\right)=\frac{n+5}{2(n+1)}$.

Proof. The vertices of $W_{1, n}$ are of two kinds: $n$ vertices which are of degree 3 will be referred to as minor vertices and the vertex of degree $n$ will be referred to as central vertex. Label the minor vertices as $v_{i}(i=\overline{1, n})$, the central vertex as $c$. There exist two cases for the shortest paths between the pairs of vertices.

Case 1 If the pair of vertices includes the central vertex and the minor vertices:

There exists only one path between those vertices that has the length as $d\left(c, v_{i}\right)=1$. Hence, the value of efficiency between these vertices is $\varepsilon\left(c, v_{i}\right)=1, \forall i=\overline{1, n}$.

Since the value of $\varepsilon\left(c, v_{i}\right)$ holds for every other $n$ pairs of vertices $W_{1, n}$, combining the efficiency values for $\forall c, v_{i} \in V\left(W_{1, n}\right)$ yields

$$
\sum \varepsilon\left(c, v_{i}\right)=n \text {. }
$$

Case 2 If the pair of vertices includes any two different minor vertices $v_{i}$ and $v_{j}$ :

We have three subcases for these minor vertices according to the length of the shortest path on the cycle between the vertices:

Subcase 1 If $d\left(v_{i}, v_{j}\right)=1$, the value of efficiency for these vertices is $\varepsilon\left(v_{i}, v_{j}\right)=$ 1. Since the value of $\varepsilon\left(v_{i}, v_{j}\right)$ holds for every other $n$ pairs of vertices $W_{1, n}$, combining the efficiency values for $\forall v_{i}, v_{j} \in V\left(W_{1, n}\right)$ yields

$$
\sum \varepsilon\left(v_{i}, v_{j}\right)=n .
$$

Subcase 2 If $d\left(v_{i}, v_{j}\right)=2$, then the value of efficiency for these vertices is $\varepsilon\left(v_{i}, v_{j}\right)=1 / 2$. Since the value of $\varepsilon\left(v_{i}, v_{j}\right)$ holds for every other $n$ pairs of vertices $W_{1, n}$, combining the efficiency values for $\forall v_{i}, v_{j} \in$ $V\left(W_{1, n}\right)$ yields

$$
\sum \varepsilon\left(v_{i}, v_{j}\right)=n / 2 .
$$


Subcase 3 If $d\left(v_{i}, v_{j}\right)>2$, then there are only one path between the vertices $v_{i}$ and $v_{j}$ with length two including central vertex $c$. Hence, the value of efficiency for these vertices is $\varepsilon\left(v_{i}, v_{j}\right)=1 / 2$. Since the value of $\varepsilon\left(v_{i}, v_{j}\right)$ holds for every other $n(n-5) / 2$ pairs of vertices $W_{1, n}$, combining the efficiency values for $\forall v_{i}, v_{j} \in V\left(W_{1, n}\right)$ yields

$$
\sum \varepsilon\left(v_{i}, v_{j}\right)=n(n-5) / 4 .
$$

Consequently, by summing up the cases 1 and 2, the value of the global efficiency of $V\left(W_{1, n}\right)$ is

$$
\begin{aligned}
E_{g l o b\left(W_{1, n}\right)} & =\frac{2}{n(n+1)}\left(n+n+\frac{n}{2}+\frac{n(n-5)}{4}\right) \\
& =\frac{n+5}{2(n+1)} .
\end{aligned}
$$

The proof is completed.

Theorem 6. If $S_{1, n}$ is a star graph with $n+1$ vertices, then the global efficiency of the star graph is $E_{\text {glob }}\left(S_{1, n}\right)=\frac{n+3}{2(n+1)}$.

Proof. The vertices of $S_{1, n}$ are of two kinds: one vertex of degree $n$ and $n$ vertices of degree one. The vertices of degree one will be referred to as minor vertices as $v_{i}(i=\overline{1, n})$ and vertex of degree $n$ to as center vertex as $c$. There exist two cases for the shortest paths between the pairs of vertices.

Case 1 If $d\left(v_{i}, v_{j}\right)=1$, then this case exists for only the pairs of vertices including the central vertex and the minor vertices. Hence, the value of efficiency for these vertices is $\varepsilon\left(c, v_{i}\right)=1 \forall i=\overline{1, n}$. Since the value of $\varepsilon\left(c, v_{i}\right)$ holds for every other $n$ pairs of vertices $S_{1, n}$, combining the efficiency values for $\forall c, v_{i} \in V\left(S_{1, n}\right)$ yields

$$
\sum \varepsilon\left(c, v_{i}\right)=n
$$

Case 2 If $d\left(v_{i}, v_{j}\right)=2$, then this case exists for only the pairs of vertices including the different two minor vertices. Hence, the value of efficiency for these vertices is $\varepsilon\left(v_{i}, v_{j}\right)=1 / 2, \forall i, j=\overline{1, n}, i \neq j$. Since the value of $\varepsilon\left(v_{i}, v_{j}\right)$ holds for every other $\left(\begin{array}{l}n \\ 2\end{array}\right)=n(n-1) / 2$ pairs of vertices $S_{1, n}$, combining the efficiency values for $\forall c, v_{i} \in V\left(S_{1, n}\right)$ yields

$$
\sum \varepsilon\left(c, v_{i}\right)=n(n-1) / 4
$$


Consequently, by summing up the cases 1 and 2, the value of the global efficiency of $S_{1, n}$ is

$$
\begin{aligned}
E_{g l o b}\left(S_{1, n}\right) & =\frac{2}{n(n+1)}\left(n+\frac{n(n-1)}{4}\right) \\
& =\frac{n+3}{2(n+1)}
\end{aligned}
$$

The proof is completed.

\section{Conclusion}

The global efficiencies of graphs discussed in the paper are given in Table 1.

Table 1: The global efficiency values of some graphs

\begin{tabular}{|c|c|c|c|}
\hline Graph & Vertex Number & Edge Number & Global Efficiency \\
\hline$C_{n}$ cycle graph & $n$ & $n$ & $\left\{\begin{array}{l}\frac{1}{(n-1)\lfloor n / 2\rfloor}, \text { if } n \text { is odd } \\
\frac{2}{(n-1)\lfloor n / 2\rfloor} \text { if } n \text { is } \text { even }\end{array}\right.$ \\
\hline$K_{n}$ complete graph & $n$ & $\left(\begin{array}{l}n \\
2\end{array}\right)=\frac{n(n-1)}{2}$ & $\frac{n+5}{2(n+1)}$ \\
\hline$W_{1, n}$ wheel graph & $n+1$ & $2 n$ & $\frac{n+3}{2(n+1)}$ \\
\hline$S_{1, n}$ star graph & $n+1$ & $n$ & \multicolumn{2}{|c|}{} \\
\hline
\end{tabular}

The line diagram for $n=\overline{1,25}$ is gained above Figure 1 by using the values obtained in Table 1. Accordingly, it is possible to make a comparison between some known special graph types discussed in the paper.

A comparison is made in order to decide which type is more stable with respect to the line diagram obtained in Figure 1. According to the data obtained, it can be observed that the order for the graphs from more stable to less stable is complete graph, wheel, star, cycle and path. 
Figure 1: The comparison about the global efficiency of some special graphs

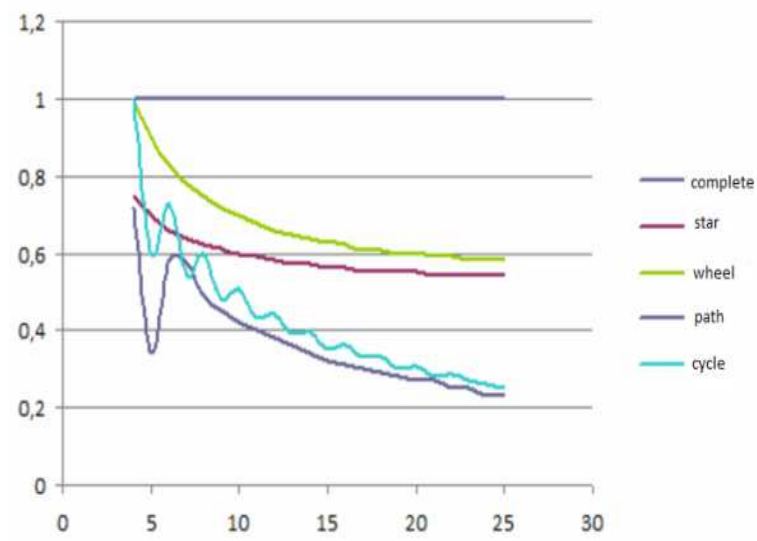

\section{References}

[1] C.A.Barefoot, R.Entringer, and H.Swart, Vulnerability in Graphs-A Comparative Survey. J.Comb.Math.Comb.Comput. 1, (1987), 13-22.

[2] Boccaletti S., Buldu J.,Criado R.,Flores J.,Latora V.,Pello J. and Romance M., The Structure and Dynamics of Complex Networks, Chaos 17, 043110 (2007).

[3] Boccaletti S., Criado R.,Pello J., Romance M. and Vela-Perez M., Vulnerability and fall of efficiency in complex networks: A new approach with computational advantages, Int. J. Bifurcation Chaos 19,727-735(2009).

[4] Bondy J.A. and Murty U.S.R., Graph theory with applications, American Elsevier Publishing Co., Inc., New York(1976).

[5] Chartrand G., Leisnak L., Graphs and Digraphs, Second Edition, Wadsworth.Monterey (1986)

[6] V.Chvatal, Tough Graphs and Hamiltonian Circuits., Discrete Math., 5, (1973), 215-228.

[7] Crucitti P., Latora V., Marchiori M. and Rapisarda A., Error and attacktolerance of complex networks, Physica A 340, 388-394 (2004). 
[8] Ek B.,Caitlin V. and Narayan D.A., Efficiency of star-like graphs and the Atlanta subway network,Phisica A 392,5481-5489(2013).

[9] Jung H. A., On a class of posets and the corresponding comparability graphs, Journal of Combinatorial Theory, Series B 24(2) (1978) 125-133.

[10] Latora V. and Marchiori M., Efficient behavior of small-world networks, Phys.Rev. Lett. 87,198701,2001.

[11] Latora V. and Marchiori M., A measure of centrality based on network efficiency, New Journal of Phys. 9,188(2007).

[12] West D.B., Introduction to Graph Theory, Prentice Hall, NJ(2001).

[13] Woodall D.R., The binding number of a graph and its Anderson number, J. Combin. Theory Ser. B 15 (1973) 225-255. 